\title{
Zygmund's Type Inequality to the Polar \\ Derivative of A Polynomial
}

\author{
M.S. Pukhta \\ SKUAST-Kashmir, Srinagar 190025, India \\ mspukhta_67@yahoo.co.in
}

Keywords: Polynomials, Zygmund's inequality, polar derivative.

Abstract. In this paper we improve a result recently proved by Irshad et al. [On the Inequalities Concerning to the Polar Derivative of a Polynomial with Restricted Zeroes, Thai Journal of Mathematics, 2014 (Article in Press)] and also extend Zygmund's inequality to the polar derivative of a polynomial.

\section{Introduction}

Let $P(z)$ ba a polynomial of degree $n$, then

$$
\max _{|z|=1}\left|P^{\prime}(z)\right| \leq n \max _{|z|=1}|P(z)|
$$

inequlaity (1) is a well known result of S. Bernstein [1]. Equality holds in (1) if and only if $P(z)$ has all its zeros at the origin.

Inequality (1) was extended to $L_{p}$-norm $p \geq 1$ by Zygmund [2], who proved that if $P(z)$ is a polynomial of degree $n$, then

$$
\left\{\frac{1}{2 \pi} \int_{0}^{2 \pi}\left|P^{\prime}\left(e^{i \theta}\right)\right|^{p} d \theta\right\}^{\frac{1}{p}} \leq n\left\{\frac{1}{2 \pi} \int_{0}^{2 \pi}\left|P\left(e^{i \theta}\right)\right|^{p} d \theta\right\}^{\frac{1}{p}}
$$

Equality holds in (2) for $P(z)=\alpha z^{n},|\alpha| \neq 0$. If we let $p \rightarrow \infty$ in (2), we get inequlaity (1).

Let $\alpha$ be a complex number. If $P(z)$ is a polynomial of degree $n$, then the polar derivative of $P(z)$ with respect to the point $\alpha$, denoted by $D_{\alpha} P(z)$, is defined by

$$
D_{\alpha} P(z)=n P(z)+(\alpha-z) P^{\prime}(z)
$$

clearly $D_{\alpha} P(z)$ is a polynomial of degree at most $n-1$ and it generalizes the ordinary derivative in the sense that

$$
\lim _{\alpha \rightarrow \infty}\left[\frac{D_{\alpha} P(z)}{\alpha}\right]=P^{\prime}(z) .
$$

As an extension of (1) to the polar derivative, Aziz and Shah [3], have shown that if $P(z)$ is a polynomial of degree $n$, then for every real or complex number $\alpha$ with $|\alpha|>1$ and for $|z|=1$,

$$
\left|D_{\alpha} P(z)\right| \leq n|\alpha| \max _{|z|=1}|P(z)|
$$

As a generalization of (2) to the polar derivaative Aziz et al. [4], proved the following result. 
Theorem A If $P(z)$ is a polynomial of degree $n$, then for every complex number $\alpha$ with $|\alpha| \geq 1$ and $p \geq 1$

$$
\left\{\int_{0}^{2 \pi}\left|D_{\alpha} P\left(e^{i \theta}\right)\right|^{p} d \theta\right\}^{\frac{1}{p}} \leq n(|\alpha|+1)\left\{\int_{0}^{2 \pi}\left|P\left(e^{i \theta}\right)\right|^{p} d \theta\right\}^{\frac{1}{p}}
$$

For the Class of polynomials having no zeros in $|z|<1$, inequality (2) was improved by D-Bruijin [5] that if $P(z) \neq 0$ in $|z|<1$, then for $p \geq 1$

$$
\left\{\int_{0}^{2 \pi}\left|P^{\prime}\left(e^{i \theta}\right)\right|^{p} d \theta\right\}^{\frac{1}{p}} \leq n C_{p}\left\{\int_{0}^{2 \pi}\left|P\left(e^{i \theta}\right)\right|^{p} d \theta\right\}^{\frac{1}{p}}
$$

where

$$
c_{p}=\left\{\frac{1}{2 \pi} \int_{0}^{2 \pi}\left|1+e^{i \theta}\right|^{p} d \theta\right\}^{-\frac{1}{p}}
$$

As an extension to the polar derivative. A. Aziz and N. Rather [6], proved the following generalization of (5). In fact they proved.

Theorem B If $P(z)$ is a polynomial of degree $n$ which does not vanish in $|z|<1$, then for every complex number $\alpha$ with $|\alpha| \geq 1$ and $p \geq 1$

$$
\left\{\int_{0}^{2 \pi}\left|D_{\alpha} P\left(e^{i \theta}\right)\right|^{p} d \theta\right\}^{\frac{1}{p}} \leq n(|\alpha|+1) c_{p}\left\{\int_{0}^{2 \pi}\left|D_{\alpha} P\left(e^{i \theta}\right)\right|^{p} d \theta\right\}^{\frac{1}{p}}
$$

where $C_{p}$ is defined by (7).

Recently, Irshad et al. [7] proved the following result.

Theorem $\mathbf{C}$ If $P(z)$ is a polynomial of degre $n$ which does not vanish in $|z|<K \leq 1$, then for every $\alpha, \beta \in C$ with $|\alpha| \geq K,|\beta| \leq 1$ and $p \geq 1$

$$
\begin{aligned}
& \left\{\int_{0}^{2 \pi}\left|e^{i \theta} D_{\alpha} P\left(e^{i \theta}\right)+n \frac{(|\alpha|-K)}{K+1} \beta P\left(e^{i \theta}\right)\right|^{p} d \theta\right\}^{\frac{1}{p}} \\
& \quad \leq n\left(1+|\alpha|+2 \frac{(|\alpha|-K)}{K+1}|\beta|\right) C_{p}\left\{\int_{0}^{2 \pi}\left|P\left(e^{i \theta}\right)\right|^{p} d \theta\right\}^{\frac{1}{p}}
\end{aligned}
$$

where $C_{p}$ is defined by (7).

In this paper we prove the following more general result which also generalize Theorem B and yields a number of known polynomial inequalities. 
Theorem 1. If $P(z)=a_{n} z^{n}+\sum_{j=\mu}^{n} a_{n-j} z^{n-j}, 1 \leq \mu \leq n$ be a polynomial of degree $n$ which does not vanish in $|z|<K \leq 1$, then for every $\alpha, \beta \in C$ with $|\alpha| \geq K,|\beta| \leq 1$ and $p \geq 1$

$$
\begin{aligned}
& \left\{\int_{0}^{2 \pi}\left|e^{i \theta} D_{\alpha} P\left(e^{i \theta}\right)+n \frac{\left(|\alpha|-K^{\mu}\right)}{K^{\mu}+1} \beta P\left(e^{i \theta}\right)\right|^{p} d \theta\right\}^{\frac{1}{p}} \\
& \leq n\left(1+|\alpha|+2 \frac{\left(|\alpha|-K^{\mu}\right)}{K^{\mu}+1}|\beta|\right) C_{p}\left\{\int_{0}^{2 \pi}\left|P\left(e^{i \theta}\right)\right|^{p} d \theta\right\}^{\frac{1}{p}}
\end{aligned}
$$

where $C_{p}$ is defined by (7), or equivalently

$$
\begin{aligned}
& \left\|e^{i \theta} D_{\alpha} P\left(e^{i \theta}\right)+n \frac{\left(\left|\alpha-K^{\mu}\right|\right)}{K^{\mu}+1} \beta P\left(e^{i \theta}\right)\right\|_{p} \\
& \quad \leq n\left(1+|\alpha|+2 \frac{\left(|\alpha|-K^{\mu}\right)}{K^{\mu}+1}|\beta|\right) \frac{\left\|P\left(e^{i \theta}\right)\right\|_{p}}{\left\|1+e^{i \phi}\right\|_{p}}
\end{aligned}
$$

Remark. If we choose $\mu=1$ in (10), we get Theorem $\mathrm{C}$ and if we choose $\beta=0$ and $K=1$ in (10), we get Theorem B.

\section{Lemmas}

For the proof of this theorem, we need the following lemmas. The first lemma is due to Gulshan Singh et al. [8].

Lemma 1. Let $P(z)=a_{n} z^{n}+\sum_{j=\mu}^{n} a_{n-j} z^{n-j}, 1 \leq \mu \leq n$ be a polynomial of degree having all its zeros in the disk $|z| \leq K, K \leq 1$, then for every real or complex number $\alpha$ with $|\alpha| \geq K, K \leq 1$ and for $|z|=1$

$$
\left|D_{\alpha} P(z)\right| \geq n\left(\frac{|\alpha|-K^{\mu}}{K^{\mu}}\right)|P(z)|
$$

Lemma 2. Let $Q(z)$ be a polynomial of degree $n$ having all its zeros in $|z|<K, K \leq 1$ and $P(z)$ is a polynomial of degree not exceeding that of $Q(z)$. If $|P(z)| \leq|Q(z)|$ for $|z|=K \leq 1$, then for every $\alpha, \beta \in C$ with $|\alpha| \geq K, \beta \mid \leq 1$

$$
\left|z D_{\alpha} P(z)+n \frac{\left(|\alpha|-K^{\mu}\right)}{K^{\mu}+1} \beta P(z)\right| \leq\left|z D_{\alpha} Q(z)+n \frac{\left(|\alpha|-K^{\mu}\right)}{K^{\mu}+1} \beta Q(z)\right|
$$

Proof. Since $|\lambda P(z)| \leq|P(z)| \leq|Q(z)|$, for $\lambda<1$ and $|z|=K$, then for Rouche's Theorem $Q(z)-\lambda P(z)$ and $Q(z)$ have the same number of zeros in $|z|<K$. On the other hand by inequality $|P(z)| \leq|Q(z)|$ for $|z|=K$, any zero of $Q(z)$, that lies on $|z|=K$, in the zero of $P(z)$. Therefore, $Q(z)-\lambda P(z)$ has all its zero in the closed disk $|z| \leq K$. Hence by Lemma 1 for all real or complex numbers $\alpha$ with $|\alpha| \geq K$ and $|z|=1$, we have

$$
\left|z D_{\alpha}(Q(z)-\lambda P(z))\right| \geq n \frac{\left(|\alpha|-K^{\mu}\right)}{K^{\mu}+1}|Q(z)-\lambda P(z)|
$$

Now consider a similar argument that for any value of $\beta$ with $|\beta|<1$, we have

$$
\left|z D_{\alpha}(Q(z)-\lambda P(z))\right| \geq n\left(\frac{|\alpha|-K^{\mu}}{K^{\mu}+1}\right)|Q(z)-\lambda P(z)|
$$




$$
>n|\beta|\left(\frac{|\alpha|-K^{\mu}}{K^{\mu}+1}\right)|Q(z)-\lambda P(z)|
$$

where $|z|=1$, resulting in

$$
T(z)=\left|z D_{\alpha} Q(z)-\lambda z D_{\alpha} P(z)\right|+n \beta \frac{|\alpha|-K^{\mu}}{K^{\mu}+1}\{Q(z)-\lambda P(z)\} \neq 0
$$

where $|z|=1$.

That is

$$
T(z)=\left|z D_{\alpha} Q(z)+n \beta \frac{|\alpha|-K^{\mu}}{K^{\mu}+1} Q(z)\right|-\lambda\left|z D_{\alpha} P(z)+n \beta \frac{|\alpha|-K^{\mu}}{K^{\mu}+1} P(z)\right| \neq 0
$$

for $|z|=1$

We also conclude that

$$
\left|z D_{\alpha} Q(z)+n \beta \frac{|\alpha|-K^{\mu}}{K^{\mu}+1} Q(z)\right| \geq\left|z D_{\alpha} P(z)+n \beta \frac{|\alpha|-K^{\mu}}{K^{\mu}+1} P(z)\right|
$$

for $|z|=1$.

If (16) is not true, then there is a point $z=z_{0}$ with $\left|z_{0}\right|=1$, such that

$$
\left|z_{0} D_{\alpha} Q\left(z_{0}\right)+n \beta \frac{|\alpha|-K^{\mu}}{K^{\mu}+1} Q\left(z_{0}\right)\right|<\left|z_{0} D_{\alpha} P\left(z_{0}\right)+n \beta \frac{|\alpha|-K^{\mu}}{K^{\mu}+1} P\left(z_{0}\right)\right|
$$

Take

$$
\lambda=\frac{z_{0} D_{\alpha} Q\left(z_{0}\right)+n \beta \frac{|\alpha|-K^{\mu}}{K^{\mu}+1} Q\left(z_{0}\right)}{z_{0} D_{\alpha} P\left(z_{0}\right)+n \beta \frac{|\alpha|-K^{\mu}}{K^{\mu}+1} P\left(z_{0}\right)}
$$

then $|\lambda|<1$ with this choice, we have from (15), $T\left(z_{0}\right)=0$ for $\left|z_{0}\right|=1$. But this contradicts the fact that $T(z) \neq 0$ for $|z|=1$. For $\beta$ with $|\beta|=1$, (16) follows by continuity.

This completes the proof.

The next lemma is due to Aziz and Rather [4].

Lemma 3. If $P(z)$ is a polynomial of degree $n$ such that $P(0) \neq 0$ and $Q(z)=z^{n} p\left(\frac{1}{\bar{z}}\right)$, then for every $p \geq 0$ and $\phi$ real

$$
\int_{0}^{2 \pi} \int_{0}^{2 \pi}\left|Q^{\prime}\left(e^{i \theta}\right)+e^{i \theta} P^{\prime}\left(e^{i \theta}\right)\right|^{p} d \theta d \phi \leq n^{p} \int_{0}^{2 \pi}\left|P\left(e^{i \theta}\right)\right|^{p} d \theta
$$

\section{Proof of the theorem}

Proof of the Theorem. Let $P(z)$ be a polynomial of degree $n$ which does not vanish in $|z|<K$, $K \leq 1$. By Lemma 2 for complex numbers $\alpha$, $\beta$ with $|\alpha| \geq K,|\beta| \leq 1$, we have

$$
\left|z D_{\alpha} P(z)+n \frac{\left(|\alpha|-K^{\mu}\right)}{K^{\mu}+1} \beta P(z)\right| \leq\left|z D_{\alpha} Q(z)+n \frac{\left(|\alpha|-K^{\mu}\right)}{K^{\mu}+1} \beta Q(z)\right|
$$


For every real $\phi$ and $\xi \geq 1$, we have

$$
\left|\xi+e^{i \theta}\right| \geq\left|1+e^{i \phi}\right|
$$

which implies for any $p \geq 0$

$$
\left\{\int_{0}^{2 \pi}\left|\xi+e^{i \phi \mid}\right|^{p} d \phi\right\}^{\frac{1}{p}} \geq\left\{\int_{0}^{2 \pi}\left|1+e^{i \phi}\right|^{p} d \phi\right\}^{\frac{1}{p}}
$$

If $e^{i \theta} D_{\alpha} P\left(e^{i \theta}\right)+n \frac{\left(|\alpha|-K^{\mu}\right)}{K^{\mu}+1} \beta P\left(e^{i \theta}\right) \neq 0$, we can take

$$
\xi=\frac{e^{i \theta} D_{\alpha} Q\left(e^{i \theta}\right)+n \frac{\left(|\alpha|-K^{\mu}\right)}{K^{\mu}+1} \beta Q\left(e^{i \theta}\right)}{e^{i \theta} D_{\alpha} P\left(e^{i \theta}\right)+n \frac{\left(|\alpha|-K^{\mu}\right)}{K^{\mu}+1} \beta P\left(e^{i \theta}\right)}
$$

where according to (19), $|\xi| \geq 1$. Now

$$
\begin{aligned}
& \int_{0}^{2 \pi} \mid e^{i \theta} D_{\alpha} Q\left(e^{i \theta}\right)+n \frac{\left(|\alpha|-K^{\mu}\right)}{K^{\mu}+1} \beta Q\left(e^{i \theta}\right) \\
& +\left.e^{i \phi}\left[e^{i \theta} D_{\alpha} P\left(e^{i \theta}\right)+n \frac{\left(|\alpha|-K^{\mu}\right)}{K^{\mu}+1} \beta P\left(e^{i \theta}\right)\right]\right|^{p} d \phi \\
& =\left|e^{i \theta} D_{\alpha} P\left(e^{i \theta}\right)+n \frac{\left(|\alpha|-K^{\mu}\right)}{K^{\mu}+1} \beta P\left(e^{i \theta}\right)\right| \int_{0}^{2 \pi}\left|\xi+e^{i \phi}\right|^{p} d \phi \\
& \geq\left|e^{i \theta} D_{\alpha} P\left(e^{i \theta}\right)+n \frac{\left(|\alpha|-K^{\mu}\right)}{K^{\mu}+1} \beta P\left(e^{i \theta}\right)\right| \int_{0}^{2 \pi}\left|1+e^{i \phi}\right|^{p} d \phi
\end{aligned}
$$

This inequality is trivially true if

$$
e^{i \theta} D_{\alpha} P\left(e^{i \theta}\right)+n \frac{\left(|\alpha|-K^{\mu}\right)}{K^{\mu}+1} \beta P\left(e^{i \theta}\right)=0
$$

Integrating both sides of (21) with respect to $\theta$ from 0 to $2 \pi$, we have

$$
\begin{aligned}
& \int_{0}^{2 \pi} \int_{0}^{2 \pi} \mid e^{i \theta} D_{\alpha} Q\left(e^{i \theta}\right)+n \frac{\left(|\alpha|-K^{\mu}\right)}{K^{\mu}+1} \beta Q\left(e^{i \theta}\right) \\
& \quad+\left.e^{i \phi}\left[e^{i \theta} D_{\alpha} P\left(e^{i \theta}\right)+n \frac{\left(|\alpha|-K^{\mu}\right)}{K^{\mu}+1} \beta P\left(e^{i \theta}\right)\right]\right|^{p} d \theta d \phi \\
& \geq \int_{0}^{2 \pi}\left|e^{i \theta} D_{\alpha} P\left(e^{i \theta}\right)+n \frac{\left(|\alpha|-K^{\mu}\right)}{K^{\mu}+1} \beta P\left(e^{i \theta}\right)\right|^{p} d \theta \int_{0}^{2 \pi}\left|1+e^{i \phi}\right|^{p} d \phi
\end{aligned}
$$


Now for $0 \leq \theta<2 \pi$

$$
\begin{aligned}
& \left|e^{i \theta} D_{\alpha} Q\left(e^{i \theta}\right)+n \frac{\left(|\alpha|-K^{\mu}\right)}{K^{\mu}+1} \beta Q\left(e^{i \theta}\right)+e^{i \phi}\left[e^{i \theta} D_{\alpha} P\left(e^{i \theta}\right)+n \frac{\left(|\alpha|-K^{\mu}\right)}{K^{\mu}+1} \beta P\left(e^{i \theta}\right)\right]\right| \\
& =\mid\left[e^{i \theta}\left\{n Q\left(e^{i \theta}\right)+\left(\alpha-e^{i \theta}\right) Q^{\prime}\left(e^{i \theta}\right)\right\}+n \frac{\left(|\alpha|-K^{\mu}\right)}{K^{\mu}+1} \beta Q\left(e^{i \theta}\right)\right] \\
& +e^{i \phi}\left[e^{i \theta}\left\{n P\left(e^{i \theta}\right)+\left(\alpha-e^{i \theta}\right) P^{\prime}\left(e^{i \theta}\right)\right\}+n \frac{\left(|\alpha|-K^{\mu}\right)}{K^{\mu}+1} \beta P\left(e^{i \theta}\right)\right] \mid \\
& =\mid\left[e^{i \theta}\left\{n Q\left(e^{i \theta}\right)-e^{i \theta} Q^{\prime}\left(e^{i \theta}\right)\right\}+\alpha e^{i \theta} Q^{\prime}\left(e^{i \theta}\right)+n \frac{\left(|\alpha|-K^{\mu}\right)}{K^{\mu}+1} \beta Q\left(e^{i \theta}\right)\right] \\
& \quad+e^{i \phi}\left[e^{i \theta}\left\{n P\left(e^{i \theta}\right)-e^{i \phi} P^{\prime}\left(e^{i \theta}\right)\right\}+\alpha e^{i \theta} P^{\prime}\left(e^{i \theta}\right)+n \frac{\left(|\alpha|-K^{\mu}\right)}{K^{\mu}+1} \beta P\left(e^{i \theta}\right)\right] \mid
\end{aligned}
$$

Since $Q(z)=z^{n} P\left(\frac{1}{\bar{z}}\right)$, we have $P(z)=z^{n} Q \overline{\left(\frac{1}{\bar{z}}\right)}$ and it can be easily verified that for $0 \leq \theta<2 \pi$

$$
n P\left(e^{i \theta}\right)-e^{i \theta} P^{\prime}\left(e^{i \theta}\right)=e^{i(n-1) \theta} \overline{Q^{\prime}\left(e^{i \theta}\right)}
$$

and

$$
n Q\left(e^{i \theta}\right)-e^{i \theta} Q^{\prime}\left(e^{i \theta}\right)=e^{i(n-1) \theta} \overline{P^{\prime}\left(e^{i \theta}\right)}
$$

From (24)

$$
\begin{aligned}
& \left|e^{i \theta} D_{\alpha} Q\left(e^{i \theta}\right)+n \frac{\left(|\alpha|-K^{\mu}\right)}{K^{\mu}+1} \beta Q\left(e^{i \theta}\right)+e^{i \phi}\left[e^{i \theta} D_{\alpha} P\left(e^{i \theta}\right)+n \frac{\left(|\alpha|-K^{\mu}\right)}{K^{\mu}+1} \beta P\left(e^{i \theta}\right)\right]\right| \\
& =\mid\left[e^{i \theta}\left\{e^{i(n-1) \theta} \overline{P^{\prime}\left(e^{i \theta}\right)}\right\}\right]+\alpha e^{i \theta}\left[Q^{\prime}\left(e^{i \theta}\right)+e^{i \phi} P^{\prime}\left(e^{i \theta}\right)\right] \\
& \quad+n \frac{\left(|\alpha|-K^{\mu}\right)}{K^{\mu}+1} \beta\left[Q\left(e^{i \theta}\right)+e^{i \phi} P\left(e^{i \theta}\right)\right]+e^{i \phi} e^{i \theta} e^{i(n-1) \theta} Q^{\prime}\left(e^{i \theta}\right) \mid
\end{aligned}
$$

Therefore, (22) in conjunction with (25) gives

$$
\begin{aligned}
& \left\{\int_{0}^{2 \pi} \int_{0}^{2 \pi} \mid e^{i \theta} e^{i(n-1) \theta}\left\{\overline{P^{\prime}\left(e^{i \theta}\right)}+e^{i \phi} \overline{Q^{\prime}\left(e^{i \theta}\right)}\right\}+\alpha e^{i \phi}\left[Q^{\prime}\left(e^{i \theta}\right) e^{i \phi} P^{\prime}\left(e^{i \theta}\right)\right]\right. \\
& \left.+\left.n \frac{\left(|\alpha|-K^{\mu}\right)}{K^{\mu}+1} \beta\left[Q\left(e^{i \theta}\right)+e^{i \phi} P\left(e^{i \theta}\right)\right]\right|^{p} d \theta d \phi\right\}^{\frac{1}{p}} \\
& \geq\left\{\int_{0}^{2 \pi}\left|e^{i \theta} D_{\alpha} P\left(e^{i \theta}\right)+n \frac{\left(|\alpha|-K^{\mu}\right)}{K^{\mu}+1} \beta P\left(e^{i \theta}\right)\right|^{p} d \theta \int_{0}^{2 \pi}\left|1+e^{i \phi}\right|^{p} d \phi\right\}^{\frac{1}{p}}
\end{aligned}
$$


By Minkowski inequality, we have

$$
\begin{gathered}
\left\{\int_{0}^{2 \pi}\left|e^{i \theta} D_{\alpha} P\left(e^{i \theta}\right)+n \frac{\left(|\alpha|-K^{\mu}\right)}{K^{\mu}+1} \beta P\left(e^{i \theta}\right)\right|^{p} d \theta \int_{0}^{2 \pi}\left|1+e^{i \phi}\right|^{p} d \phi\right\}^{\frac{1}{p}} \\
\geq\left\{\int_{0}^{2 \pi} \int_{0}^{\frac{1}{p}}\left|Q^{\prime}\left(e^{i \theta}\right)+e^{i \phi} P^{\prime}\left(e^{i \theta}\right)\right|^{p} d \theta d \phi\right\}^{2 \pi}\{1+|\alpha|\} \\
+\left|n \frac{\left(|\alpha|-K^{\mu}\right)}{K^{\mu}+1} \beta\right|\left\{\int_{0}^{2 \pi} \int_{0}^{2 \pi}\left|Q\left(e^{i \theta}\right)+e^{i \phi} P\left(e^{i \theta}\right)\right|^{p} d \theta d \phi\right\}^{\frac{1}{p}}
\end{gathered}
$$

By Lemma 3, we have

$$
\begin{aligned}
& \left\{\int_{0}^{2 \pi}\left|e^{i \theta} D_{\alpha} P\left(e^{i \theta}\right)+n \frac{\left(|\alpha|-K^{\mu}\right)}{K^{\mu}+1} \beta P\left(e^{i \theta}\right)\right|^{p} d \theta \int_{0}^{2 \pi}\left|1+e^{i \phi}\right|^{p} d \phi\right\}^{\frac{1}{p}} \\
& \leq\left\{2 n^{p} \pi \int_{0}^{2 \pi}\left|P\left(e^{i \theta}\right)\right|^{p} d \theta\right\}^{\frac{1}{p}}\{1+|\alpha|\} \\
& \quad+2 n\left|\frac{\left(|\alpha|-K^{\mu}\right)}{K^{\mu}+1}\right|\left\{2 \pi \int_{0}^{2 \pi}\left|P\left(e^{i \theta}\right)\right|^{p} d \theta\right\}^{\frac{1}{p}} \\
& =\left[n(1+|\alpha|)+2 n \frac{\left(|\alpha|-K^{\mu}\right)}{K^{\mu}+1}|\beta|\right]\left\{2 \pi \int_{0}^{2 \pi}\left|P\left(e^{i \theta}\right)\right|^{p} d \theta\right\}^{\frac{1}{p}}
\end{aligned}
$$

This implies

$$
\begin{aligned}
& \left\{\int_{0}^{2 \pi}\left|e^{i \theta} D_{\alpha} P\left(e^{i \theta}\right)+n \frac{\left(|\alpha|-K^{\mu}\right)}{K^{\mu}+1} \beta P\left(e^{i \theta}\right)\right|^{p} d \theta\right\}^{\frac{1}{p}} \\
& \leq n\left(1+|\alpha|+2 \frac{\left(|\alpha|-K^{\mu}\right)}{K^{\mu}+1}|\beta|\right) C_{p}\left\{\int_{0}^{2 \pi}\left|P\left(e^{i \theta}\right)\right|^{p} d \theta\right\}^{\frac{1}{p}}
\end{aligned}
$$

where $C_{p}$ in defined by (7),

or equivalently,

$$
\begin{aligned}
& \left\|e^{i \theta} D_{\alpha} P\left(e^{i \theta}\right)+n \frac{\left(|\alpha|-K^{\mu}\right)}{K^{\mu}+1} \beta P\left(e^{i \theta}\right)\right\| \\
& \quad \leq n\left(1+|\alpha|+2 \frac{\left(|\alpha|-K^{\mu}\right)}{K^{\mu}+1}|\beta|\right) \frac{\left\|P\left(e^{i \theta}\right)\right\|_{p}}{\left\|1+P\left(e^{i \phi}\right)\right\|_{p}}
\end{aligned}
$$

Hence the result. 


\section{References}

[1] S. Bernstein, Lecons sur Less propries extréxtremaleset la meilleure dune fonctions, (Paris, 1926).

[2] A. Zygmund, A remark on conjugate series, Proc. London Math. Soc. 34(2) (1932), 392-400.

[3] A. Aziz and W.M. Shah, Inequalities for the polar derivaive of a polynomial, Indian J. Pure Appl. Math., 29 (1998), 163-173.

[4] A. Aziz and N.A. Rather, Some Zygmunds type $L^{p}$-inequlaity for polynomial, J. Math. Anal. Appl., 289 (2004), 14-29.

[5] N.G. De-Bruijin, Inequalitis concerning polynomials in the complex domain, Nedrel. Akad. Weternsch.Proc. Ser. A., 50 (1947), 1265-1272; Indag. Math., 9 (1947), 591-598.

[6] A. Aziz and N.A. Rather, On an inequality concerning the polar derivative of a poly, Proc. Indian Acad. Sci. (Math. Sci.), 117(3) (August 2003), 349-357.

[7] Irshad Ahmed, A. Liman and W.M. Shah, On the inequalities concering to the polar derivative of a polynomial with restricted zeros, Thai Journal of Mathematics, (2014).

[8] G. Singh, W.M. Shah and A. Liman, A generalized inequality for the polar deriviative of polynomial, Journal of Inequalities and Applicaitons, 2013 2013:183. 